\title{
Subjective memory and strategy use in mild cognitive impairment and healthy aging
}

\author{
Paula Schimidt Brum, Mônica Sanches Yassuda, and Orestes Vicente Forlenza \\ Universidade de São Paulo, São Paulo, SP, Brazil
}

\begin{abstract}
Limited information is available about subjective memory and strategy use in seniors with mild cognitive impairment (MCI). We investigated whether differences exist in the perception of changes in memory, perceived frequency of forgetting, overall memory evaluation, and strategy use between seniors with MCI and unimpaired older adults. The study included 56 participants, aged 60 years and older, including 28 normal controls (NC) and 28 MCI patients. The participants completed the Short Cognitive Performance Test, the Story and Grocery list recall tasks, the 15-item Geriatric Depression Scale, the Memory Complaint Questionnaire for the perception of changes in episodic memory, the McNair Frequency of Forgetting Questionnaire, and a single question that evaluated overall memory. The Bousfield semantic clustering measure was calculated to assess semantic clustering for list recall. The number of underlined words during story encoding was calculated to assess strategy use. Participants with MCI had significantly worse scores on Story and Grocery list recall, semantic clustering, and overall memory evaluation. No differences were found in the number of underlined words. List recall was significantly correlated with semantic clustering in both groups (NC: $r=.58, p=.001$; MCI: $r=.57, p=.002$ ). Participants with MCI appeared to be less efficacious when using memory strategies, which may be associated with poor memory performance. Keywords: semantic clustering, strategy use, subjective memory, awareness, mild cognitive impairment, aging.
\end{abstract}

Received 2 March 2013; received in revised form 11 April 2013; accepted 20 April 2013. Available online 27 June 2013.

\section{Introduction}

Mild cognitive impairment (MCI) refers to a transitional stage between age-associated memory impairment and dementia. The recent diagnostic criteria for MCI attributable to Alzheimer's disease (AD) suggest that MCI should encompass concern about possible cognitive decline by the patient, informant, or clinician, evidence of cognitive impairment in at least one domain, independence in functional activities, and not meeting the criteria for dementia (Albert et al., 2011). Agreement has been reached that MCI consists of multiple subtypes including amnestic and non-amnestic, with impairment in single or multiple domains (Winblad et al., 2004; Petersen, 2011). Much evidence suggests that patients with amnestic MCI are more likely to convert to $\mathrm{AD}$ than patients with other

Paula Schimidt Brum and Orestes Vicente Forlenza, Departamento e Instituto de Psiquiatria, Faculdade de Medicina, Universidade de São Paulo, São Paulo, SP, Brazil. Mônica Sanches Yassuda, Gerontologia, Escola de Artes, Ciências e Humanidades (EACH), Universidade de São Paulo, São Paulo, SP, Brazil. Correspondence regarding this article should be directed to: Orestes Vicente Forlenza, MD, $\mathrm{PhD}$, Departamento e Instituto de Psiquiatria, Faculdade de Medicina, Universidade de São Paulo, Ovídio Pires de Campos, 785, São Paulo, SP, 05403-010, Brazil. Phone/Fax: +55-11-3069-7267. E-mail: pabrum@usp.br; yassuda@usp. br; forlenza@usp.br
MCI subtypes (Mariani, Monastero, \& Mecocci, 2007; Forlenza, Diniz, Nunes, Memória, Yassuda, \& Gattaz, 2009; Hanyu, 2010).

Although MCI patients exhibit lower memory performance, limited information is available about how they approach cognitive tasks. Malek-Ahmadi, Raj, \& Small (2011) and Ribeiro, Guerreiro, \& De Mendonça (2007) suggested that MCI patients tend to use less semantic clustering spontaneously during word list recall tasks. However, results from the latter study indicated that older adults with MCI benefited from using semantic cues. Bennett, Golob, Parker, \& Starr (2006) reported that older adults with $\mathrm{MCI}$ and normal controls (NC) did not differ in a measure of recall strategy (i.e., subjective organization and recall consistency), although MCI patients exhibited significantly lower performance in list recall. Froger, Taconnat, Landré, Beigneux, \& Isingrini (2009), in a small but elegant study, reported that older adults with MCI used semantic cues during encoding, but performance benefits were observed only in tasks that used recognition paradigms.

Hutchens et al. (2012) studied strategy use and strategy knowledge in adults with amnestic MCI and healthy seniors. The participants completed questionnaires to assess strategy knowledge and the self-reported use of internal and external strategies in everyday life. Additionally, strategy use (i.e., serial, subjective, and semantic clustering) was directly 
observed in measures of retrospective and prospective memory (i.e., taking notes of tasks to be completed). The researchers found decreased strategy knowledge and decreased use of semantic clustering in the amnestic MCI group but equivalent self-reported use of strategies (i.e., internal and external) and observed use of external strategies. Semantic clustering was associated with retrospective memory performance in both groups and prospective memory in the amnestic MCI group only. Previous studies, therefore, appear to suggest that seniors with MCI are less strategic with regard to internal strategies such as semantic encoding. However, the use of strategies is associated with better recall in this group. Group differences are not usually found for self-reported strategy use and the observed use of external strategies, but these findings are preliminary and confirmational studies are needed.

The awareness of memory problems in MCI represents a relatively underinvestigated topic, with the extant studies yielding conflicting results. Chung \& Man (2009), for example, reported that the self-appraisal of cognitive function in persons with MCI did not correlate with informant reports or neuropsychological test scores, but self-reported strategy use correlated with list recall and everyday memory tests in their sample. These findings suggest that older adults with MCI may show signs of diminished awareness of their subtle impairments in cognitive functioning.

Orfei et al. (2010) investigated the presence of anosognosia (i.e., lack of awareness of cognitive impairment) among patients with MCI and AD. The lack of awareness was higher and more frequent in $\mathrm{AD}$ patients $(42 \%)$ and rare in MCI patients $(3 \%$ among multiple-domain $\mathrm{MCI}$ ). The decreased awareness of deficits was associated with reduced verbal episodic memory scores in the MCI group.

In a recent study, Zamboni et al. (2013) investigated awareness of the characteristics of the self (i.e., cognitive, behavioral, and physical) and of a caregiver or close friend in patients with AD and MCI and healthy seniors using a discrepancy paradigm. Alzheimer's disease patients - but not MCI patients - demonstrated impaired self-awareness but no impairment in appraising the characteristics of others. Imaging results suggested that lack of awareness was associated with reduced activation of the medial prefrontal cortex and anterior temporal lobe.

In a systematic review of subjective memory complaints in MCI that included 16 studies, Roberts, Clare, \& Woods (2009) found significant variability in the level of awareness of cognitive impairment in seniors with MCI who may either under- or overestimate their impairment.

Therefore, the literature to date is inconclusive with regard to strategy use and awareness of memory problems in older adults with MCI. It is unknown whether older adults with MCI use memory strategies such as semantic encoding more intensely than unimpaired individuals to compensate for their decline or, alternatively, whether they fail to use them because of limited cognitive processing resources. The association between memory performance and strategy use also warrants further investigation because it may vary in these two groups considering that MCI patients may need to rely more intensely on memory strategies to maintain previous levels of performance. Additionally, understanding strategy use in MCI may have clinical implications. Decreased strategy use may contribute to the diagnosis of MCI or identification of higher risk for converting to AD. Examining deficits in awareness in MCI is also relevant because lack of awareness of cognitive deficits may prevent the person from seeking diagnosis and treatment.

We designed the present study to investigate whether strategy use differences exist in episodic memory tasks, assessed directly in the study protocol at encoding and retrieval between MCI patients and unimpaired older adults. We were also interested in testing for group differences in the perception of memory decline, frequency of forgetting, and general memory evaluation.

We hypothesized that the MCI group would show decreased strategy use and episodic memory performance. Based on previous studies, we expected the MCI group to show awareness of deficits and indicate a higher perception of change, higher frequency of forgetting, and poor memory evaluation. This hypothesis was derived from the fact that one of the diagnostic criteria for MCI is to have memory complaints.

\section{Methods \\ Participants}

This study was part of a larger research project on the neurobiology of AD and MCI that investigates markers of risk and prognosis. Normal controls and MCI patients were recruited from the Psychogeriatric Outpatient Unit of the Institute of Psychiatry, University of São Paulo. All participants were independent, lived in their own homes, and went to the Institute of Psychiatry when called for evaluation. They were assessed by a multidisciplinary team of psychiatrists, neuropsychologists, and geriatricians. Diagnoses were reached through consensus discussions. Petersen, Smith, Waring, Ivnik, Tangalos, \& Kokmen (1999) criteria were used for the MCI diagnosis. The 56 participants included in the present study were classified as NC ( $n$ $=28)$ or MCI $(n=28)$. Among the MCI patients, 13 had single-domain amnestic MCI and 15 had multipledomain amnestic MCI.

Patients in this unit undergo a vast battery of psychometric instruments biannually that assess cognitive and functional skills. The battery includes the Cambridge Examination for Mental Disorders of the Elderly (CAMCOG; Paradela, Lopes, \& Lourenço, 2009), Informant Questionnaire on Cognitive Decline in the Elderly (IQCODE; Jorm, \& Jacomb, 1989; Sanchez \& Lourenço, 2009), Rivermead Behavioral Memory 
Test (RBMT; Yassuda et al., 2010), Fuld Object Memory Evaluation (FOME; Fuld, Masur, Blau, Crystal, \& Aronson, 1990), Short Cognitive Performance Test (SKT; Flaks, Forlenza, Pereira, Viola, \& Yassuda, 2009), Nelson's Modified Card Sorting Test (Nelson, 1976), Trail Making tests A and B (Hamdan \& Hamdan, 2009), and Vocabulary and Block Design subtests of the Wechsler Adult Intelligence Scale, revised (WAIS-R; Nascimento \& Figueiredo, 2002).

The participants also underwent laboratory exams to detect any treatable causes of cognitive decline. Neuroimaging exams were performed when the team deemed them necessary.

Inclusion criteria for this study were the following: $>60$ years of age and diagnosis of MCI in the past 6 months or regarded as cognitively unimpaired. Exclusion criteria were the following: diagnosis of other psychiatric and neurological disorders and presence of sensory or motor limitations that could hinder participation.

With regard to medication use, both groups (MCI and NC) were on similar drug regimens. The participants were taking medications for hypertension (16 in the $\mathrm{MCI}$ group and 12 in the $\mathrm{NC}$ group) and diabetes (six in the MCI group and nine in the NC group). The use of other drugs was reported less frequently for dyslipidemia (five in the MCI group and four in the NC group), osteoporosis (three in the MCI group and two in the NC group), rheumatism (four in the MCI group), arthrosis (seven in the NC group), gastritis (two in the MCI group), insomnia (seven in the MCI group and six in the $\mathrm{NC}$ group).

\section{Materials}

The following instruments were used in this study: SKT, Story and Grocery list recall tasks, 15-item Geriatric Depression Scale (GDS), McNair Frequency of Forgetting Questionnaire, and Memory Complaint Questionnaire (MAC-Q).

The SKT (Flaks et al., 2006, 2009) is commonly used to identify and monitor cognitive decline. The test consists of nine sub-items, three of which are related to memory and six related to attention. Scores in the attention domain range from 0 to 18 and scores in the memory domain range from 0 to 9 . Total SKT score ranges from 0 to 27 , with lower scores suggesting cognitive preservation. This scale has five different versions. Version A was used in this study. The SKT has been previously validated for use in the Brazilian population.

To assess episodic memory, Story and Grocery list recall tasks were used. For the Story recall task, the participants had to read a 64-idea story for $5 \mathrm{~min}$. After an interference task that lasted $2 \mathrm{~min}$, they were asked to write as many of the ideas as they could remember. They had $5 \mathrm{~min}$ to write down the recalled ideas. During the test instructions, the participants were told they could use a pencil to underline important ideas in the text. The number of underlined words was used as a measure of strategy use during story encoding. The story was developed by Dixon, Hultsch, \& Hertzog (1989) and adapted to Brazilian Portuguese in a validation study by Yassuda, Lasca, \& Neri (2005). This memory test has been used extensively in the cognitive aging literature (e.g., Talib, Yassuda, Diniz, Forlenza, \& Gattaz, 2008; West, Bagwell, \& Dark-Freudeman, 2008; Hastings \& West, 2009; Yassuda, Batistoni, Fortes, \& Neri, 2006).

For the Grocery list recall task, the participants were asked to study a list of 35 grocery items for $5 \mathrm{~min}$. Shortly afterward, they were asked to write down as many of the items as they could remember in $3 \mathrm{~min}$. The list could be divided into seven categories with five items each, such as vegetables, fruits, meats, and cleaning supplies. The scores ranged from 0 to 35 items. This test allows the calculation of the Bousfield semantic clustering measure (Bousfield \& Puffs, 1965; Carvalho, Neri, \& Yassuda, 2010) in which the number of items recalled within semantic categories is divided by the total number of items recalled minus one. It ranges from 0 to 1 , with higher scores indicating a higher degree of categorization during the recall of the list items. This list was developed by West \& Thorn (2001) and adapted to Brazilian Portuguese in a validation study by Yassuda et al. (2005). This memory test has been used extensively in the cognitive aging literature (e.g., Talib et al., 2008; West et al., 2008; Yassuda et al., 2006).

The short version of the GDS containing 15 questions was used (Almeida \& Almeida, 1999; Paradela et al., 2009). Participants with GDS scores $>10$ were excluded from the analyses.

The MAC-Q (Crook, Feher, \& Larrabee, 1992; Mattos, Lino, Rizo, Alfano, Araújo, \& Raggio, 2003) was used to assess the participants' perception of changes in their memory ability. The participants were asked to compare their current memory performance on certain tasks such as remembering names and a shopping list with their memory performance when they were 1820 years old. The participants had to choose between "much better" (1), "a bit better" (2), "the same" (3), "a bit worse" (4), and "much worse" (5). Scores $\geq 25$ are usually interpreted as indicative of the presence of memory complaints.

The McNair Frequency of Forgetting Questionnaire (McNair \& Kahn, 1983; Paulo \& Yassuda, 2010) was used to assess the frequency of perceived memory failure. A list of 26 possible memory failures was shown to participants, and they were asked to indicate how often these events happened. For example, the participants were asked, "How often do you forget to pay bills?" They could respond "rarely" (0), "sometimes" (1), "often" (2), and "always" (3). The scores ranged from 0 to 72. Higher scores suggest a higher frequency of selfreported memory failure.

A single question was used to capture the participants' overall evaluation of their memory: "How do you evaluate your memory today?" Possible answers varied from "awful" (0) to "excellent" (5), and higher scores indicated a better perception of memory. 


\section{Procedures}

All participants signed an informed consent form previously approved by the institution's ethics committee. Assessments lasted $\sim 1 \mathrm{~h}$ and were conducted in groups of three patients by a neuropsychologist who was unaware of their clinical diagnosis. Most of the instruments were completed by the patients under the instruction and supervision of the neuropsychologist. The SKT was applied individually after the group procedures were concluded.

\section{Statistical analysis}

Kolmogorov-Smirnov tests indicated that most of the variables did not present a normal distribution. Therefore, nonparametric tests were used. The MannWhitney test for independent samples was used to compare NC and MCI groups in strategy use and memory performance. Spearman correlations were used to assess associations between strategy use and memory performance and correlations among other study variables. Cohen's $d$ scores were calculated as a measure of effect size. Data were analyzed using SPSS for Windows, v.14, and the level of significance adopted for the statistical tests was .05.

\section{Results}

This study involved 56 participants: 28 normal controls (NC) and 28 with MCI. The NC group was comprised of 17 women, and there were 19 women in the MCI group. Sample characteristics are presented in Table 1. Diagnostic groups were statistically comparable with regard to age, education, and number of depressive symptoms. As expected, significant differences in SKT scores were found between groups.

As shown in Table 1, significant group differences were found in Story and Grocery list recall and semantic clustering with higher scores in the NC group. However, no significant differences were found in the number of underlined words during story encoding, the perception of decline in memory performance, or reports of the frequency of forgetting events. However, the MCI group expressed a more negative view of their memory in the overall memory evaluation.

Spearman correlations indicated that Grocery list recall performance was significantly correlated with the degree of semantic clustering in the $\mathrm{NC}$ group $(r=.58, p=.001)$ and MCI group $(r=.57, p=.002)$. No significant correlation was found between the number of underlined words during story encoding and number of recalled ideas $(r=.02, p=.90$ for $\mathrm{NC} ; r=.20, p=$ .29 for MCI).

With regard to awareness of changes in memory, a significant positive correlation was found between SKT attention scores and MAC-Q scores in the $\mathrm{NC}$ group $(r=.40, p=.03)$, suggesting that worse performance in attention may be associated with a higher perception of change. In the MCI group, a negative correlation was found between Story recall and overall memory evaluation $(r=-.43, p=.02)$.

\section{Discussion}

The present study compared older patients with MCI and unimpaired seniors with regard to episodic memory performance (Story and Grocery list recall), use of mnemonic strategies (semantic clustering in Grocery list recall and underlining words in Story recall), and certain aspects of subjective memory (perception of change, perceived frequency of forgetting, and overall memory evaluation). Patients with MCI had lower performance on Story and Grocery list recall, used less semantic clustering, and reported lower overall memory ability. Nevertheless, they had comparable scores for the number of underlined words, perceived change in

Table 1. Means and standard deviations for sample characteristics, objective and subjective memory measures in NC and MCI groups

\begin{tabular}{lcccc}
\hline & NC (n=28) & MCI (n=28) & p value & Cohen's d \\
\hline Age & $72.43(3.87)$ & $74.77(6.63)$ & 0.11 & -0.42 \\
Schooling & $15.93(3.12)$ & $14.38(3.86)$ & 0.08 & 0.45 \\
GDS & $3.03(1.76)$ & $3.31(1.39)$ & 0.39 & -0.23 \\
SKT Total & $1.75(1.66)$ & $3.92(2.31)$ & $\mathbf{0 . 0 0}$ & -0.96 \\
Grocery list recall & $15.60(5.23)$ & $11.75(5.44)$ & $\mathbf{0 . 0 0}$ & 0.68 \\
Story recall & $24.96(7.87)$ & $16.14(8.81)$ & $\mathbf{0 . 0 0}$ & 0.93 \\
Semantic clustering* & $0.44(0.24)$ & $0.31(0.23)$ & $\mathbf{0 . 0 4}$ & 0.53 \\
Underlined words & $13.03(17.53)$ & $11.03(13.96)$ & 0.63 & 0.12 \\
MAC-Q & $27.38(6.65)$ & $24.85(6.31)$ & 0.16 & 0.37 \\
McNair & $25.71(12.47)$ & $27.32(13.46)$ & 0.64 & -0.12 \\
Memory evaluation & $2.85(0.89)$ & $2.42(0.69)$ & $\mathbf{0 . 0 4}$ & 0.52 \\
\hline
\end{tabular}

Note: $\mathrm{p}$ value refers to Mann Whitney's test for independent samples, *Bousfield measure. Significant results in bold. 
memory, and frequency of forgetting compared with the $\mathrm{NC}$ group.

Few published studies have investigated strategy use in MCI patients, and the available data are conflicting. Studies have suggested that MCI patients have difficulty using semantic clustering spontaneously (Ribeiro et al., 2007; Malek-Ahmadi et al., 2011; Hutchens et al., 2012). Another study (Bennett et al., 2006) suggested that MCI patients use similar memory strategies as normal controls but continue to present lower performance. Our results appear to be consistent with the former studies where we observed less semantic clustering in MCI patients. Underlining words during story encoding appeared to be irrelevant for Story recall in both groups because it did not correlate with performance.

Froger et al. (2009) recently demonstrated that older adults with MCI use and benefit from semantic cues during encoding (i.e., having to indicate whether list items are concrete or abstract words). However, such benefits were observed only in the recognition task when MCI participants presented performance similar to the NC group when they were allocated in the semantic encoding condition. During free recall, being in the semantic encoding condition did not generate performance gains in the MCI group. The authors hypothesized that older adults with MCI may need support during encoding and retrieval, similar to $\mathrm{AD}$ patients.

In the present sample, MCI patients used less semantic clustering than the NC group. Although group differences in clustering were modest in magnitude, differences in free recall were larger, which may suggest that the MCI patients failed to fully benefit from strategy use during the free recall tasks. Patients with MCI may have difficulty gaining access to information and may have better performance if cues are provided during recall, such as in recognition paradigms. Notably, however, the higher use of semantic clustering was associated with better performance in both groups.

Participants with MCI did not report a higher perception of change and frequency of forgetting, which was expected because of lower cognitive scores. This result may suggest that seniors with MCI do not accurately report cognitive difficulties or do not perceive deficits, as shown by Zamboni et al. (2013). Nevertheless, when asked to report their overall perception of their memory ability, the MCI group appeared to be more critical than the NC group. The present results appear to be consistent with the review by Roberts et al. (2009), which suggested great variability in measures of awareness of cognitive decline in MCI patients. Conflicting findings may be partially attributable to the different measures of awareness that have been used in previous studies. Certain questions or questionnaires may better capture memory complaints that, according to current MCI diagnostic criteria, support this diagnosis. Notably, however, the new diagnostic criteria for MCI (Albert et al., 2011) permit informants or skilled clinicians to provide concern about changes in cognition. These updated criteria appear to acknowledge the fact that MCI patients may not be aware of changes in their cognitive status. The present findings suggest that MCI patients have difficulty keeping track of cognitive failures and comparing their present level of performance with previous years.

The present study has limitations. First, the strategy use measure during Story recall (i.e., underlining main ideas in text) was not correlated with performance and was not informative. The participants may have randomly underlined words and ideas. Second, subjective memory variables depended on self-report. Cognitive deficits in MCI patients may have affected question comprehension and the reliability of their answers. Future studies may need to include direct performance observations and informants' perceptions. Third, the sample size may have hindered the documentation of small group differences.

\section{Conclusion}

Future studies should further investigate strategy use and awareness of cognitive deficits in MCI. Such studies should increase our knowledge of possible cognitive explanations for the lower performance observed in MCI. Understanding strategy use in MCI patients may also guide the design of cognitive interventions for this group of patients. Awareness about cognitive limitations in MCI should also be further investigated because clinicians need to know the clinical value of MCI patients' appraisals of their cognitive performance.

\section{Acknowledgements}

The present work was supported by Fundação de Amparo à Pesquisa do Estado de São Paulo (FAPESP, project no. 09/06460-8).

\section{Conflict of Interest}

None declared.

\section{References}

Albert, M. S., DeKosky, S. T., Dickson, D., Dudois, B., Feldman, H. H., Fox, N. C., ... Phelps, C. H. (2011). The diagnosis of mild cognitive impairment due to Alzheimer's disease: Recommendations from the National Institute on Aging and Alzheimer's Association workgroups on diagnostic guidelines for Alzheimer's disease. Alzheimer's and Dementia, 7(3), 270-279.

Almeida, O. P., \& Almeida, S. A. (1999). Confiabilidade da versão brasileira da Escala de Depressão em Geriatria (GDS) versão reduzida. Arquivos de Neuropsiquiatria, 57(2B), 421-426.

Bennett, I. J., Golob, E. J., Parker, E. S., \& Starr, A. (2006). Memory evaluation in mild cognitive impairment using recall and recognition tests. Journal of Clinical and Experimental Neuropsychology, 28, 1408-1422.

Bousfield, W. A., \& Puff, C. R. (1965). Determinants of the clustering of taxonomically and associatively related word pairs. Journal of General Psychology, 73, 211-221.

Carvalho, F. C. R., Neri, A. L., \& Yassuda, M. S. (2010). Episodic memory training with emphasis on categorization for older adults without dementia and depression. Psicologia Reflexão e Crítica, 23, 317-323.

Chung, J. C. C., \& Man, D. W. K. (2009). Self-appraised, informantreported, and objective memory and cognitive function in mild cognitive impairment. Dementia and Geriatric Cognitive Disorders, 27, 187-193. 
Crook, T. H., 3rd, Feher, E. P., \& Larrabee, G. J. (1992). Assessment of memory complaint in age-associated memory impairment: The MAC-Q. International Psychogeriatrics, 4(2), 165-176.

Dixon R. A., Hultsch D. F., \& Hertzog, C. (1989). A manual of 25 three-tiered structurally equivalent texts for use in aging research (series title: CRGCA technical report, no. 2). Victoria: University of Victoria and Georgia Institute of Technology.

Flaks, M. K., Forlenza, O. V., Pereira, F. S., Viola, L. F., \& Yassuda, M. S. (2009). Short cognitive performance test: diagnostic accuracy and education bias in older Brazilian adults. Archives of Clinical Neuropsychology, 24, 301-306.

Flaks, M. K., Yassuda, M. S., Regina, A. C. B., Cid, C. G., Camargo, C. H. P., Gattaz, W. F., \& Forlenza, O. V. (2006). The Short Cognitive Performance Test (SKT): a preliminary study of its psychometric properties in Brazil. International Psychogeriatrics, 18, 121-133.

Forlenza, O. V., Diniz, B. S., Nunes, P. V., Memória, C. M., Yassuda, M. S., \& Gattaz, W. F. (2009). Diagnostic transitions in mild cognitive impairment subtypes. International Psychogeriatrics, 21, 1088-1095.

Froger, C., Taconnat, L., Landré, L., Beigneux, K., \& Isingrini, M. (2009). Effects of level of processing at encoding and types of retrieval task in mild cognitive impairment and normal aging. Journal of Clinical and Experimental Neuropsychology, 31(3), 312-321.

Fuld, P. A., Masur, D. M., Blau, A. D., Crystal, H., \& Aronson, M. K. (1990). Object-memory evaluation for prospective detection of dementia in normal functioning elderly: predictive and normative data Journal of Clinical and Experimental Neuropsychology, 12, 520-528.

Hamdan, A. C., \& Hamdan, E. M. L. R. (2009). Effects of age and education level on the Trail Making Test in a healthy Brazilian sample. Psychology \& Neuroscience, 2, 199-203.

Hanyu, H. (2010). Concept and clinical presentation of mild cognitive impairment. Brain Nerve, 62(7), 719-725.

Hastings, E. C., \& West R. L. (2009). The relative success of a selfhelp and a group-based memory training program for older adults. Psychology and Aging, 24(3), 586-594.

Hutchens, R. L., Kinsella, G. J., Ong, B., Pike, K. E., Parsons, S., Storey, E., ... Clare, L. (2012). Knowledge and use of memory strategies in amnestic mild cognitive impairment. Psychology and Aging, 27(3), 768-777.

Jorm, A. F., \& Jacomb, P. A. (1989). The Informant Questionnaire on Cognitive Decline in the Elderly (IQCODE): Socio-demographics correlates, reliability, validity and some norms. Psychological Medicine, 19, 1015-1022.

Malek-Ahmadi, M., Raj A., \& Small, B. J. (2011). Semantic clustering as a neuropsychological predictor for amnestic-MCI. Aging, Neuropsychology, and Cognition, 18(3), 280-292.

Mariani, E., Monastero, R., \& Mecocci, P. (2007). Mild cognitive impairment: a systematic review. Journal of Alzheimer's Disease, 12(1), 23-35.

Mattos, P., Lino, V., Rizo, L., Alfano, A., Araújo, C., \& Raggio, R. (2003). Memory complaints and test performance in healthy elderly persons. Arquivos de Neuro-Psiquiatria, 61(4), 920-924.

McNair, M., \& Kahn, R. (1983). Self-assessment of cognitive deficits In: T. Crook, S. Ferris, \& R. Bartus (Eds.), Assessment in geriatric psychopharmacology (pp. 137-144). New Canaan: Mark Powley Associates.

Nascimento, E., \& Figueiredo, V. L. M. (2002). WISC-III e WAISIII: alterações nas versões originais americanas decorrentes das adaptações para uso no Brasil. Psicologia Reflexão e Crítica, 15, 603-612.
Nelson, H. E. (1976). A modified card sorting test sensitive to frontal lobe defects. Cortex, 12, 313-324.

Orfei, M. D., Varsi, A. E., Blundo, C., Celia, E., Casini, A. R., Caltagirone, C., \& Spalletta, G. (2010). Anosognosia in mild cognitive impairment and mild Alzheimer's disease: Frequency and neuropsychological correlates. American Journal of Geriatric Psychiatry, 18(12), 1133-1140.

Paradela, E. M. P., Lopes, C. S., \& Lourenço, R. A. (2009). Transcultural adaptation of the Cambridge Cognitive ExaminationRevised in a public geriatric outpatient clinic. Caderno de Saúde Pública, 25(12), 2562-2570.

Paulo, D. L. V., \& Yassuda, M. S. (2010). The relation between memory complaints in the elderly and education, cognitive performance, and symptoms of depression and anxiety. Revista de Psiquiatria Clínica, 37(1), 23-26.

Petersen, R. C. (2011). Mild cognitive impairment. New England Journal of Medicine, 364, 2227-2234.

Petersen, R. C., Smith, G. E., Waring, S. C., Ivnik, R. J., Tangalos, E. G., \& Kokmen, E. (1999). Mild cognitive impairment: Clinical characterization and outcome. Archives of Neurology, 56, 303-308.

Ribeiro, F., Guerreiro, M., \& De Mendonça, A. (2007). Verbal learning and memory deficits in Mild Cognitive Impairment. Journal of Clinical and Experimental Neuropsychology, 29(2), 187-197.

Roberts, J. L., Clare, L., \& Woods, R. T. (2009). Subjective memory complaints and awareness of memory functioning in mild cognitive impairment: A systematic review. Dementia and Geriatric Cognitive Disorders, 28(2), 95-109.

Sanchez, M. A. S., \& Lourenço, R. A. (2009). Informant Questionnaire on Cognitive Decline in the Elderly (IQCODE): Adaptação transcultural para uso no Brasil. Cad. Saúde Pública [online], 25 (7), 1455-1465.

Talib, L. L., Yassuda, M. S., Diniz, B. S., Forlenza, O. V., \& Gattaz, W. F. (2008). Cognitive training increases platelet PLA2 activity in healthy elderly subjects. Prostaglandins, Leukotrienes, and Essential Fatty Acids, 78(4-5), 265-269.

West, R. L., \& Thorn, R. M. (2001). Goal-setting, self-efficacy, and memory performance in older and younger adults. Experimental Aging Research, 27(1), 41-65.

West, R. L., Bagwell, D. K., \& Dark-Freudeman, A. (2008). Selfefficacy and memory aging: the impact of a memory intervention based on self-efficacy. Aging, Neuropsychology, and Cognition, 15(3), 302-329

Winblad, B., Palmer, K., Kivipelto, M., Jelic, V., Fratiglioni, L., Wahlund, L. O., C., ... Petersen, R. C. (2004). Mild cognitive impairment - beyond controversies, towards a consensus: Report of the International Working Group on Mild Cognitive Impairment. Journal of Internal Medicine, 256, 240-246.

Yassuda, M. S., Batistoni, S. S. T., Fortes, A. G., \& Neri, A. L. (2006). Treino de memória no idoso saudável: Benefícios e mecanismos. Psicologia: Reflexão e Crítica, 19(3), 470-481.

Yassuda, M. S., Flaks, M. K., Viola, L. F., Pereira, F. S., Memoria, C. M., Nunes, P. V., \& Forlenza, O. V. (2010). Psychometric characteristics of the Rivermead Behavioural Memory Test (RBMT) as an early detection instrument for dementia and mild cognitive impairment in Brazil. International Psychogeriatrics, 22, 1003-1011.

Yassuda, M. S., Lasca, V. B., \& Neri, A. L. (2005). Metamemory and self-efficacy: A validation study of research instruments in memory aging. Psicologia Reflexão e Crítica, 18(1), 78-90.

Zamboni, G., Drazich, E., McCulloch, E., Filippini, N., Mackay, C. E., Jenkinson, M., Tracey, I., \& Wilcock, G. K. (2013). Neuroanatomy of impaired self-awareness in Alzheimer's disease and mild cognitive impairment. Cortex, 49, 668-678. 